\title{
A Web based Inventory Control System using Cloud Architecture and Barcode Technology for Zambia Air Force
}

\author{
Thomas Muyumba \\ Department of Electrical and Electronic Engineering \\ University of Zambia \\ Lusaka, Zambia
}

\author{
Jackson Phiri \\ Department of Computer Science \\ University of Zambia \\ Lusaka, Zambia
}

\begin{abstract}
Inventory management of spares is one of the activities Zambia Air Force (ZAF) undertakes to ensure optimal serviceability state of equipment to effectively achieve its roles. This obligation could only be made possible by automating the current manual and paper based inventory system. A web based inventory management system using cloud architecture and barcode technology was proposed. A literature review was conducted on three technologies used in the inventory management that is Radio Frequency Identification (RFID), Barcode Technology and Near Field Communication (NFC). A review was also undertaken on the related works to identify the concept that could be adopted in the proposed system. A baseline study was performed to understand the challenges faced by ZAF in the inventory management of spares. The results of the baseline study were analyzed and found that the challenges were attributed to the current manual inventory management system mainly due to human errors, incorrect inventory reporting and pilferage of items. The proposed prototype system was developed and tested and proved to be faster, efficient and more reliable than the manual and paper based system.
\end{abstract}

Keywords-Zambia Air Force (ZAF); inventory system; barcode technology; Radio Frequency Identification (RFID); Near Field Communication (NFC); cloud computing; web based application

\section{INTRODUCTION}

Inventory management of aircraft spares is one of the activities Zambia Air Force (ZAF) carries out to ensure that the state of the equipment is serviceable to effectively achieve its primary and secondary roles. To ensure the maintenance of equipment is up to date, ZAF procures so many types of spares that come in different sizes for various categories of aircraft as shown in Fig. 1. Among other spares that ZAF procures are Garmin Audio Control 340, Directional Gyro, Compressor Bleed, HF Receiver Exciter, HF Controller, AMP Coupler, KX165 VHF COMM/NAV, Marker Beacon Receiver, KN6ZA, Computer, Radar Altimeter, Isolation Amplifier, ATC Transponder, Pictorial NAV Indicator, Weather Radar, Transceiver, Flap Train, Access GPS, Slave Accessory, Transmitter, Slave Accessory, Radar sensor, Split Pin, Altimeter Pressure, Ignition Exciter, Indicator temperature AC Sealed Relay and High current Fuse. Some of these spares are very small in size and fuses are a good example. Therefore, it is so complex to store and track these small spares in the warehouse using a manual and paper based system. The manual and paper based system of inventory that is in place does not provide the needed efficiency and effectiveness to the maintenance of equipment. So, in order for ZAF to effectively achieve its roles, it is necessary to automate the current manual inventory business processes and adopt it for its operations as Information and Communication Technology (ICT) has become an integral component in all organizations. Most large companies and organizations in developing countries are adopting web-based applications to do their business efficiently and effectively by taking advantage of Internet presence that has rapidly spread around the world [1].

The study's focus is on computerizing inventory management processes by using cloud architecture and barcode technology. The barcode technology will make use of onedimensional barcodes, and a long-range wired barcode scanner [2], [3]. Barcode technology was preferred to other technologies such as Radio Frequency Identification (RFID) and Near Field Communication (NFC) because it is a mature technology, cheaper and simple to use.

This paper is organized as follows: The second section is literature review which covers technologies used in the inventory management and cloud computing. The third section is related works, which looks at the systems that have previously been designed and implemented to solve challenges of the similar nature. Fourth section presents the methodology. Fifth section brings out the results and the discussion of the baseline study conducted to identify challenges in the inventory management that ZAF faces, and the last section presents the conclusion.

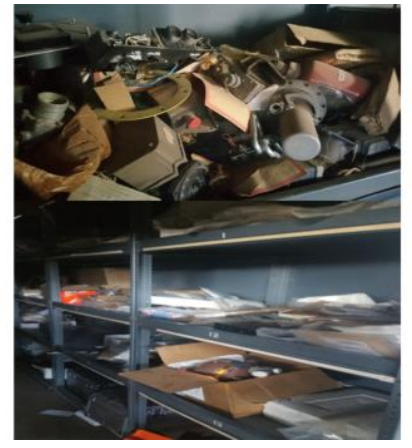

Fig. 1. Aircraft spares. 


\section{LITERATURE REVIEW}

Mwansele and Sichona [4] define inventories as dormant stocks of items that are stored in the warehouse waiting to be utilized. The management of inventory involves systems and processes that identify inventory requirements, set targets, provide replenishment techniques, report actual and projected inventory status and handle all functions related to tracking and management of material. Managers, now more than ever before, need reliable and effective inventory control to reduce costs and remain competitive [5]. According to Dobler and Burt [6], inventory alone account for as much as $30 \%$ of the organization-invested capital. Victoria and Ukpere [7] suggest that inventory control enhances profitability by reducing costs associated with storage and handling of materials. RFID, Barcode technology, NFC and related works are discussed in the subsequent sections.

\section{A. Radio Frequency Identification}

RFID is an automated system that utilizes wireless technology to uniquely identify and track tagged objects in the form of a unique serial number [8]. It gathers data about an object without the need to touch or see the data carrier. A typical RFID system is composed of four basic components including RFID tags, readers, antennas and a central node computer system which houses the database server and management software (middleware) [8]. The RFID tag or transponder is the data carrier that transmits information to the RFID reader (transceiver) within a given range through a microchip and antenna embedded in it [8], [9]. The second component in an RFID system is the reader. Its role is to query a tag and receive data from it [10]. The antenna generates radio signals to activate the tag and read or write data to it [8]. The middleware at the central node manages incorporation of data received from the reader [10]. The middleware manages the information exchange between the reader and the backend database server [10]. A computer database server mainly completes the data storage, management and read-write control of the radio frequency tags. They provide the data obtained from the reader to the software application.

The advantages of RFID include: automatic non-line-ofsight [11], [12], ability to withstand harsh environments [12], the data capacity of RFID tags permits them to vary in size, from holding only a few bits to thousands of bits [11], [12], the technology is secure [11], it provides real-time information as it is quite challenging for those organizations managing large yards to know precisely what goods and their quantity are and on which truck without first unloading the truck, which also makes it complex to direct the truck to the right drop off or parking yard location [11] and it is cost saving [12].

The disadvantages of RFID include: it is expensive compared to barcode technology and the price of RFID tags has traditionally been a significant obstacle to its widespread deployment in Supply Chain Management (SCM) [11], [12], because RFID uses the radio spectrum to transmit its signals, it is susceptible to interference that leads to the hampering of its ability to transmit clear and reliable information to RFID readers [11], it is difficult to position tags on a varying range of products to gain the most successful read rates [12], it is difficult to read multiple items when a pallet contains different items to be read, as the reader needs to be aware it is reading multiple types of items and the current RFID protocols are designed to offer the most optimal performance between readers and tags, but neglecting to address consumer privacy concerns. Privacy advocates are worried that if RFID tags are placed in common items, the product may be tracked once purchased by consumers [12].

\section{B. Barcode Technology}

The barcode technology is used in various areas of applications in computerizing the operations for the purpose of achieving efficiency, effectiveness and realizing optimal benefits from the business by scanning the inflow and outflow of barcoded items using a scanner [13]. The technology comprises of barcodes and barcode readers also known as scanners.

Barcodes are printed symbols of machine-readable data that contain information about an item to help facilitate the item's identification and tracking [13]. Sarika and Imran [2] define a barcode as an optical machine-readable representation of data that is capable of storing the physical object information in digital form to which they are attached or fixed. There are three types of barcodes that are used in enterprises: linear (1D), twodimensional (2D) and three-dimension (3D) barcodes. A linear barcode is the first generation, one-dimensional barcode that is made up of lines and spaces of various widths that create specific patterns. These patterns represent stock-keeping unit (SKU) numbers, which are easily and quickly read by computer scanners. The usage of linear barcodes is much cheaper and quite simple. Linear barcodes are widely used and they come in different types such as UPC, CodaBar, Code 25, 39, 128 and European Article Numbering (EAN) [2]. The 2Dimensional barcodes are more powerful and store more information compared to 1-Dimensional and these are in two types which include stacked 2D barcode and matrix 2D barcode [14]. The durability of 2D barcode is much high as compared to that of $1 \mathrm{D}$ barcode. Information is stored in two directions, which are horizontal as well as vertical. In 2dimensional barcodes, many thousand alphanumeric characters can be placed in a single symbol [15]. One of the most important advantages of 2D barcode is that large amount of data can be read easily and written accurately [15]. The 3dimensional barcodes are composed of an array of 3D cells, called modules, and each can be either filled or empty, corresponding to two possible values of a bit. They are just like 2D barcodes because they can contain different types of information such as pricing, height, weight and other product information. These barcodes were designed mostly to avoid the problems of high temperature, chemicals and solvents that would destroy any barcode in linear or 2-dimensional barcodes [16].

The barcode reader is an electronic tool that scans printed bar codes on items for sale or on other labels for identification purposes. It is used to extract information optically from the barcode [17].

The advantages of barcode technology include: since the main two components used to produce barcodes are paper and ink, therefore, barcodes are relatively less expensive compared to RFID technology that makes use of silicon chips [12], it is 
easy to use [11], it is scalable [12], it is reliable and accurate than manual data collection and it provides real-time information [12].

The disadvantages of barcode technology include: because barcode readers use a direct line of sight to the printed barcode when scanning a barcode, it makes the technology difficult and impractical in various industrial environments and the ordinary barcodes can only store a small amount of static information, normally around 20 characters [11].

\section{Near Field Communication}

NFC is one of the latest short-range wireless communication technologies [18]. It provides safe communication between electronic gadgets. NFC-enabled devices can just be pointed or touched by the users of their devices to other NFC-enabled devices to communicate with them. This act of communication is called tap-in or to tap and go. With NFC technology, communication is established when an NFC-compatible device is brought within a few centimeters of another which is around $20 \mathrm{~cm}$ theoretically and $4 \mathrm{~cm}$ in practical [19]. The immense benefit of the short transmission range is that it prevents eavesdropping on NFC-enabled dealings. This technology enables several innovative usage scenarios for mobile devices. It works on the basis of RFID technology that uses magnetic field induction to commence communication between electronic devices in close vicinity. Sharing of files between phones, service discovery and getting information by touching smart phones are possible applications of NFC using smart phones [18], [19]. Currently, NFC has many applications, which mostly focus on the field of Identification and authentication, ticketing systems in public transport such as trains and buses as well as contactless Electronic Point of Sales (EPOS) terminals at shopping centers. NFC has also shown promise in being used for data transfer or data beaming in applications such as smart posters or simplifying the setup of more complex communication methods such as Wi-Fi [19].

NFC technology defines two types of devices and two modes of operations. One is an initiator device and the other is the target device. The initiator device is one that initiates the communication and controls the data exchange. The target device is the one that responds to the initiator device. Active and Passive are the two operating modes of NFC. In active mode, both the initiator and the target generate the RF signal on which the data is carried. While in passive mode, only the initiator generates RF signal, and the target communicates back to the initiator using a technique called load modulation [20]. What makes the communication between the devices so easy is that, NFC protocol provides some features not found in other general-purpose protocols [21]. First of all, it is a very shortrange protocol. It supports communication at distances measured in centimeters [21]. The devices have to be literally almost touching to establish the link between them [22]. The advantage of this is that: Devices can rely on the protocol to be inherently secured since the devices must be placed very close to each other. It is easy to control the two devices to communicate by simply placing them next to each other or keeping them apart [23]. Procedure of establishing the protocol is inherently familiar to people. If you want something to communicate to, touch it. This allows for the establishment of the network connection between the devices to be completely automated and happens in a transparent manner. The whole process is fulfilled if devices recognize each other by touching and then connect to each other once touched [23]. Another important feature of this protocol is the support for the passive mode of communication [22].

\section{Cloud Computing}

Cloud computing is proving itself as an emerging technology in IT world which provides a novel business model for organizations to utilize software, applications and hardware resources without any upfront investment [24]. Cloud is a metaphor to describe web as a space where computing has been preinstalled and exist as a service; such as information, infrastructure, applications, storage and processing power on the web ready to be shared [24]. The cloud computing system consists of the front end and back end components. These components connect to each other through a network, usually the Internet. The front end is the side of the computer user or client whereas the back end is the cloud section of the system. The front end includes the client's computer or computer network and the application required to access the cloud computing system. Cloud computing comprises of three types of service models which include: Infrastructure as a Service (IaaS), Platform as Service (PaaS) and Software as a Service (SaaS). Cloud computing also comprises of four types of deployment models and these include: public, private, hybrid and community models.

In the infrastructure as a service model, the cloud providers offer cloud services such as hardware resources, storage and network infrastructure services. The virtualization is the base of this model [24]. In the platform as a service model, the cloud service providers provide application development platform for developers. They also deliver a set of APIs for developers to develop and launch their own customized applications. There is no need for them to install any development tools on their local devices and machines and the software as a service model facilitates for customers to access the applications hosted on the cloud. Instead of installing the applications on their own machines, users access these applications installed on the cloud using their own browsers [25].

The public computing model is used by the general public, which includes individuals, corporations and other types of organizations. Third party vendors essentially administer the public clouds over the Internet and services are offered on payper-use basis. The advantage of this model is that it is widely used in the development, deployment and management of enterprise applications at an affordable cost. It also allows organizations to deliver highly scalable and reliable applications rapidly at a more affordable cost. Its limitation is the security, which is a significant concern in public clouds [25]. In private computing model, the computing resources are operated exclusively by one organization. Private clouds are actually more secure than public clouds since their users are trusted individuals inside the organization. It emulates the concept of cloud computing on a private network [25]. Hybrid computing model is a composition of two or more cloud deployment models, linked in a way that data transfer takes place between them without affecting each other. This can be a combination of private, community or public clouds which are 
linked by a proprietary or standard technology that provides portability of data and applications among the composing clouds. These clouds would typically be created by the enterprise and management responsibilities would be split between the enterprise and the cloud provider. In this model, a company can outline the goals and needs of services [26]. A well-constructed hybrid cloud can be useful for providing secure services such as receiving customer payments, as well as those that are secondary to the business, such as employee payroll processing. The major drawback to the hybrid cloud is its difficult aspect in effectively creating and governing such a solution. Services from different sources must be obtained and provisioned as if they originated from a single location, and interactions between private and public components can make the implementation even more complicated [26]. The community cloud model is the type of cloud computing in which the infrastructure is shared by several organizations for a shared cause and may be managed by them or a third party service provider and it is rarely offered cloud model. These clouds are normally based on an agreement between related business organizations such as banking or educational organizations. A cloud environment operating according to this model may exist locally or remotely. The best example of a community cloud is the Facebook [26].

\section{E. Related Works}

Chandrasekharan et al. [27] developed an integrated barcode system for event management to ensure smooth and quick registrations of participants, real time stocktaking of consumables and providing exclusive secured venue-access during events.

Mathaba et al. [28] developed an inventory control system using an integration of Internet of Things (IoT) with RFID technology and web 2.0 technologies for identifying stock levels on shelves, loss prevention and as an enabler for locating misplaced stock, anti-counterfeiting of stock, stock validity and many others.

Boyinbode and Akinyede [10] developed an RFID Inventory Control system for Nigerian supermarkets to effectively detect and capture response signals transmitted from the RFID tags attached on each item that passes through the scanning zone.

Jamal et al. [29] developed a cloud computing system in which data from the scanning system is provided to the Electric Product Code (EPC) Information System that is implemented on cloud as SaaS (Software as a Service). The transmitted data is stored and managed on the cloud and is made available in a reliable manner to any application that requests it.

Some of the solutions provided by applications in the related works would be of great benefit if adopted in the inventory management of aircraft spares in ZAF. The concept of barcode technology and cloud architecture implemented in the related works would be adopted in the design of the proposed prototype. The rationale of using barcode technology is to keep track of how much stock is going out, how much remains on shelves and in the warehouse, giving commanders a real time picture of what is happening. The system would ensure that the institution does not hold much stock than is necessary in order to avoid unnecessary incurring of holding cost. The idea behind optimum stock level is to ensure that the cost is kept as low as possible.

\section{METHODOLOGY}

\section{A. Baseline Study}

The purpose of the baseline study was to establish the challenges faced by ZAF with regards to inventory management of spares. A Mixed Methods Research Methodology was used in this study.

1) Study Population: The target population for the study included ZAF employees who work under stores management, Senior Officers in charge of procurement, aircraft engineers and technicians.

2) Sample Size and Sampling Procedure: The study was conducted in three (03) Zambia Air Force Bases located in three different provinces, namely, Lusaka, Southern and Central. The three ZAF Bases were purposively sampled because the target was personnel who had the knowledge and experience about the information of interest by virtue of them working in the warehouses and proving aircraft maintenance.

3) Research Instrument: The research instruments were tailored with the sole purpose of meeting the objective of the baseline study. The instruments included the following:

- Questionnaire for ZAF employees who work in the warehouses or stores.

- Questionnaire for ZAF employees who provide aircraft maintenance.

- Interview guide for ZAF employees.

4) Data Collection: The researcher was granted authority by ZAF Command to collect both quantitative and qualitative data from ZAF employees with regards to inventory management of aircraft spares. Questionnaires were distributed to 45 respondents in the affected bases and they were given ample time to respond to the questions for qualitative data collection. The interviews were equally conducted to gather quantitative data. The whole process of data collection was done in two weeks' time.

5) Data Analysis: The quantitative data that was gathered by use of questionnaires was analyzed using IBM Statistical Package for the Social Sciences (SPSS). The results were presented in the form of tables and charts.

\section{B. System Design}

The system requirements specification and model design phase of the research study employed the use of qualitative data from interviews that ZAF personnel supplied. The interviews with the stores personnel and personnel who service the equipment provided the qualitative data needed to come up with the current business process and thereafter design the automated inventory business process and the system architecture.

1) Current Business Process: The current business process is shown in Fig. 2. It is derived from interviews that were 
conducted. In the business process, Aircraft Maintenance Unit (AMU) provides maintenance to equipment to ensure that it is in a serviceable state. When a particular spare part on the equipment is defective, it is indicated on Form 700 and forwarded to Technical Control to make a demand. The Officer Commanding Technical Control raises an internal demand to technical stores on ZAF Form 674 also called Internal Demand and Issue Voucher. The details that are required to be filled on this form include: part number, description of the equipment, purpose for which the spare part is required, quantity and the inventory number. The technical stores personnel issue out the spare part when it is available in stores on Form 674. If the spare part is not available, an external demand is raised to source for the missing item from Central Equipment Depot (CED). The baseline study revealed that the current process of managing inventory of aircraft spares makes extensive use of forms.

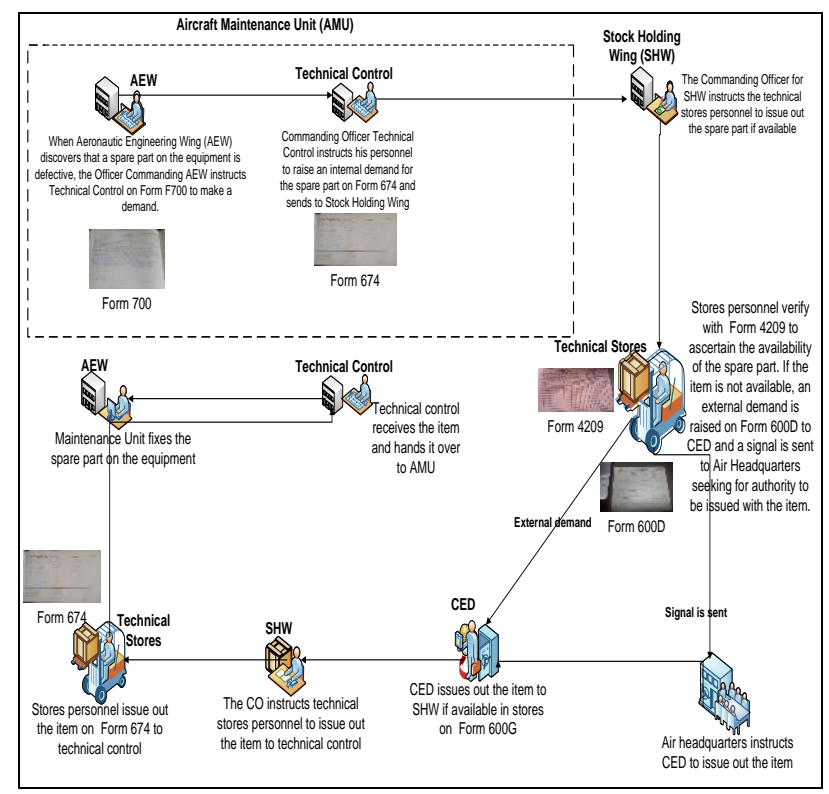

Fig. 2. Current business process.

2) Proposed Business Process: The proposed automated demand business process depicted in Fig. 3 is derived from the current demand business process methodologies as described by the stores personnel in Fig. 2. Automating manual and paper based phases in the processes is the change that is proposed here. Barcode technology was a preferred technology to be used in the proposed inventory business process because it is a mature technology, relatively cheaper, easy to implement and simple to use compared to radio frequency identification and near field communication.

The centralized database stores all the information about the spares, suppliers and employees. The application links the barcode readers to the centralized database server. The spares that are received in the warehouse already come with barcodes. Once the barcode reader scans the item, the barcode transmits information to the scanner and the scanner reads out the information and transmits the information read from the barcode to the software. The application then interprets the numbers from the barcode and then writes the information in the database. Equally, when issuing out the spares the barcode reader scans the item and transmits the information read from the barcode to the application. The application then interprets the numbers from the barcode and matches those numbers with the information in the database indicating the type of spares they represent. For instance, if the information is for a fuse for category $826 \mathrm{Y}$, it will be able to show its description and there will be an instant reduction in the number of fuses for category $826 \mathrm{Y}$ in the database. If the minimum number of fuses in that category is reached, the system will automatically send a message to the management to reorder that particular item. This allows the stores personnel to track which spares have been issued out with a handheld scanner. Specialized software keeps track of how much stock is going out the door via issuance and how much remains on shelves and in the warehouse, giving managers a real time picture of what is happening. The software analyzes the data and makes recommendations for reordering strategies. The Closed Circuit Television (CCTV) is incorporated in the proposed business process to monitor how business is conducted in the stores.

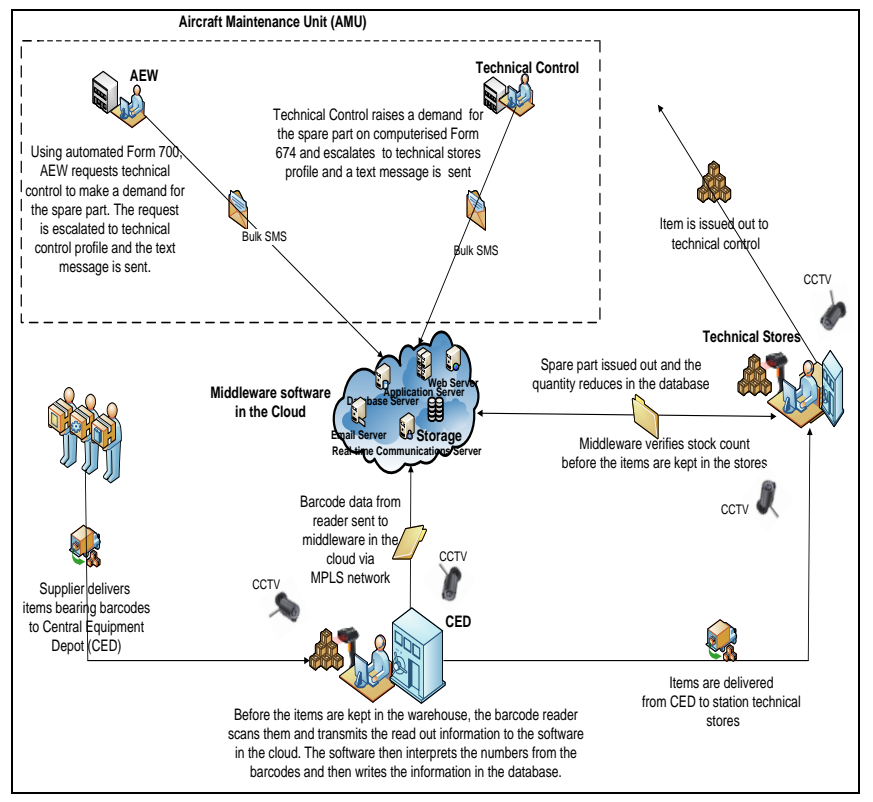

Fig. 3. Proposed business process.

3) Proposed System Architecture: The proposed system architecture utilizes the private cloud infrastructure and barcode technology to manage inventory of aircraft spares via the web interface. As the baseline study showed that the current system is tedious hence the proposed model would help reduce costs and time it takes to conduct business. The proposed system architecture is shown in Fig. 4.

ZAMTEL, the largest telecommunications company in Zambia provides the MPLS network while ZAF provides the private cloud services. The MPLS backbone comprises of fiber and microwave. ZAF exclusively operates computing resources in the cloud. There are a number of servers installed in the cloud such as Email server, Database server, Application server, Web server, Real-time communication server and other 
services, like Data storage and Backup storage. The rationale herein is to provide services to remote bases without the hassles of the hardware, software and security of information. This kind of cloud computing is more secure than public clouds since their users are trusted individuals inside the organization. It allows users to have the benefits of cloud computing without some of the pitfalls. ZAF would have complete control over how data is managed and what security measures are to be put in place. This leads to users having more confidence and control over the system.

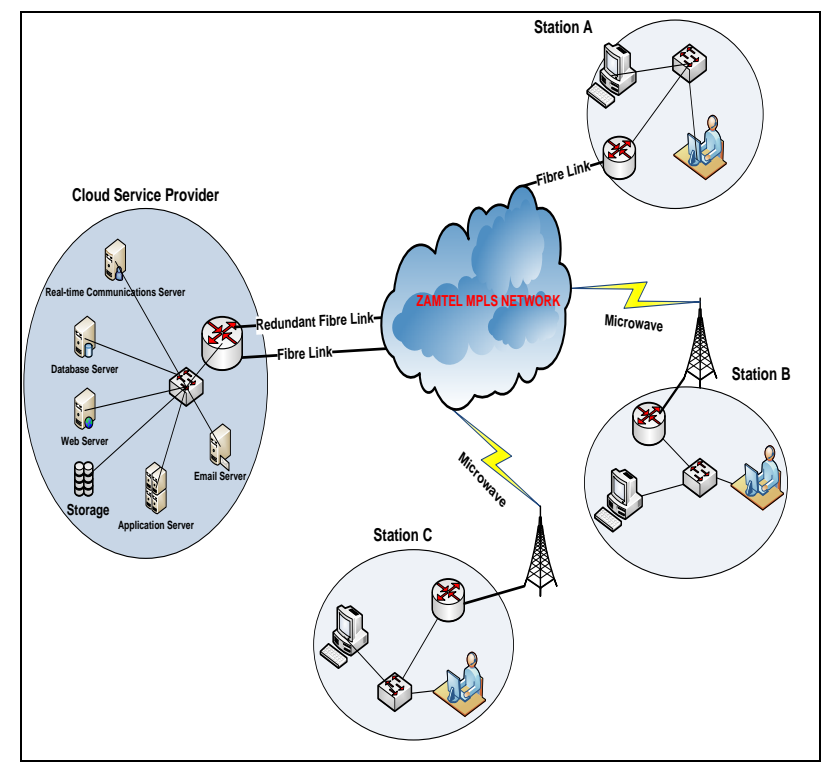

Fig. 4. Proposed system architecture.

\section{Use Cases}

The Use Case diagrams in Fig. 5 shows the interactions between the actors and the system in the system implementation. The main actors of the system were the middleware, reader and application users. The barcode reader is responsible for recording inventory stock items by scanning barcodes attached to items and sending data read to the middleware. The barcode middleware sits between the barcode reader and the backend database. It is responsible for managing exchange of information between the reader and the backend system. The users at the application interface first log into the system and then perform transactions, view inventory data and generate reports.

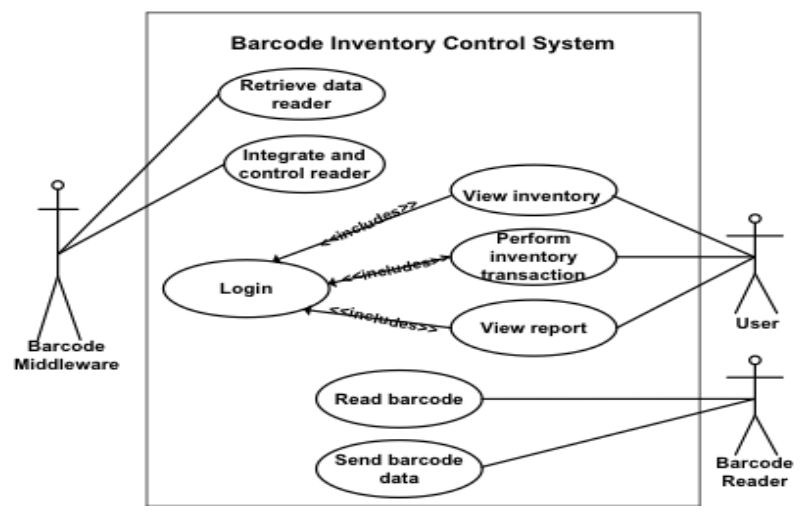

Fig. 5. Use case diagrams.

\section{Sequence Diagram}

Fig. 6 shows a sequence diagram for the "Read Barcode" from Reader Use Cases. The reader firstly monitors barcode activity. The user interface (UI) starts and then the control object is instantiated. The control object reads the barcodes as the stock items are scanned. Upon reading data from barcodes, the reader retrieves the data and sends to middleware for processing.

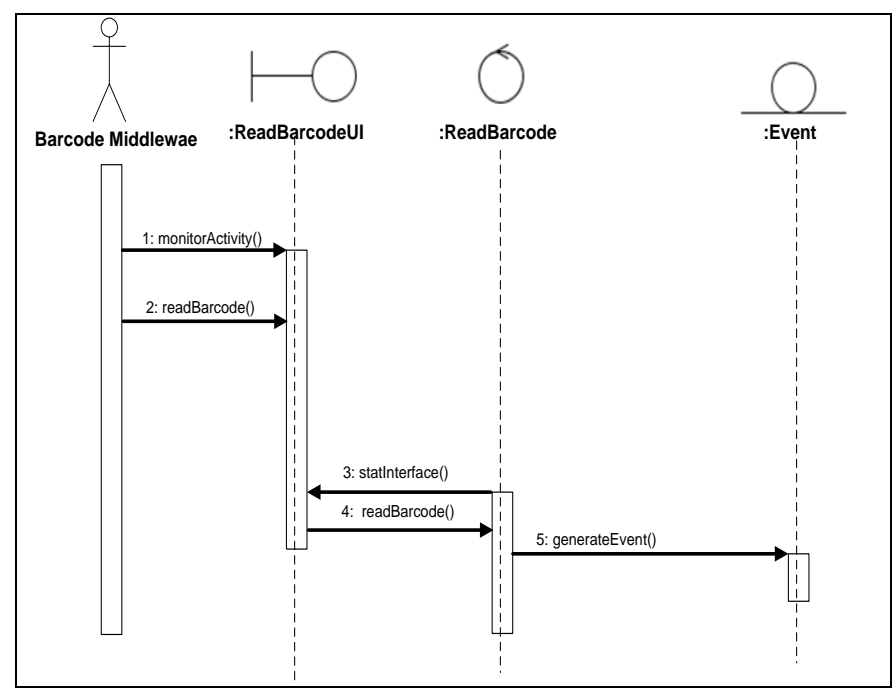

Fig. 6. Sequence diagram.

\section{E. Class Diagram}

The class diagram notations describe the structure of the proposed system by indicating system's classes, and their attributes, operations or methods, and the relationships among the classes. The class diagram in Fig. 7 illustrates the common components of classes, class attributes and class operations.

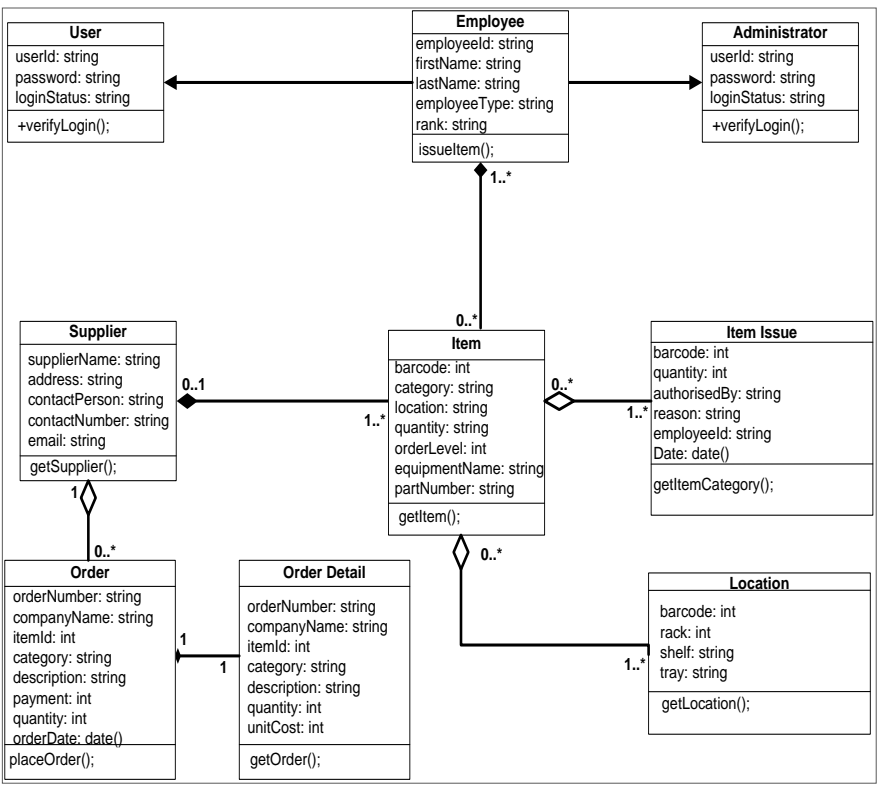

Fig. 7. Class diagram. 


\section{F. Entity Relationship Diagram}

Fig. 8 shows an Entity Relationship (ER) model diagram for the barcode inventory system. The ER model provides a representation of user reality. It consists of entities, attributes and relationships that have been reasonably assumed for the proposed system. The assumptions are derived from the requirements that have been gathered from the survey and interviews.

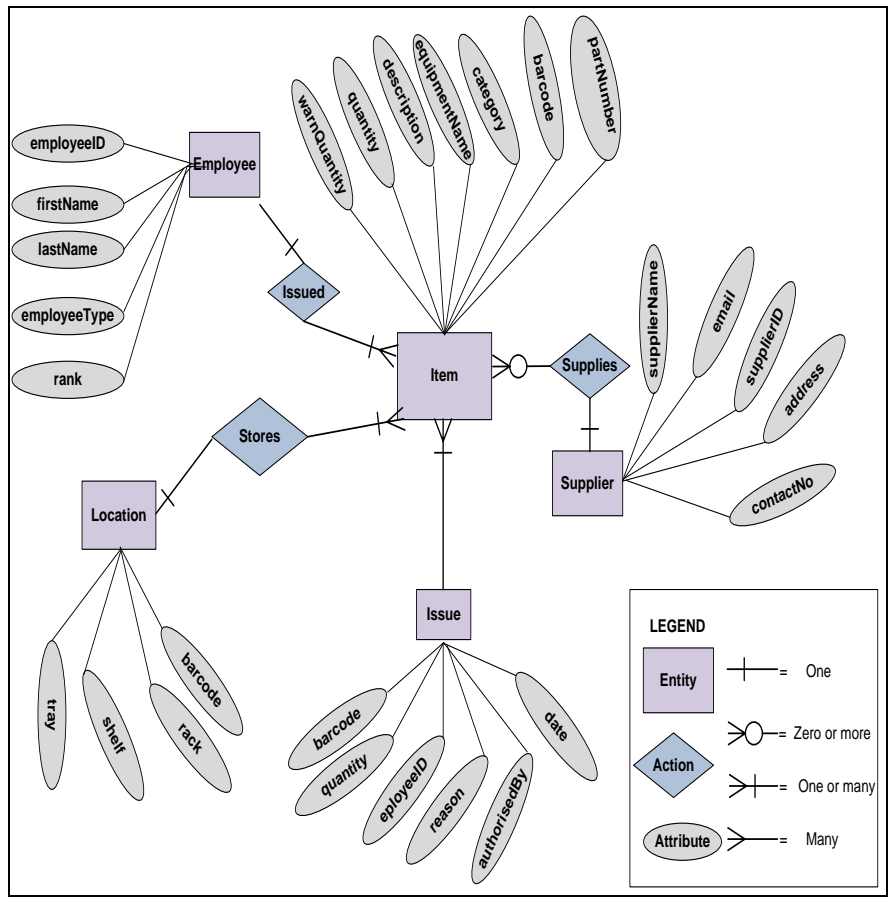

Fig. 8. Entity relationship diagram.

\section{G. System Prototype Development}

The system consists of frontend and backend components. The frontend was developed using HyperText Markup Language (HTML) for formatting of text in the document, JavaScript for interactivity in the web pages and Cascade Style Sheet (CSS), which provides the look and feel of the web pages. The backend is the server-side component of the system. The system runs on apache webserver and the database is designed using MySQL, which is a structured query language. The software was developed using Hypertext Processor (PHP) programming language for dynamic web applications.

\section{RESULTS}

The results obtained from the baseline study and system prototype development and testing are presented in this section. The main purpose of conducting the baseline study was to ascertain the challenges that ZAF faces regarding inventory management of aircraft spares. The proposed prototype application was developed show as proof of concept of how the fully implemented system would work to alleviate the challenges currently faced by ZAF.

1) Baseline Study: The data collected from the baseline study was analyzed using descriptive statistics and the results were presented in form of charts. When the respondents were asked if verification of stock count is done physically, 79\% agreed and $21 \%$ were not sure. When asked if there was any loss of service during business time, $61 \%$ reported that there was loss of service due to wrong inventory reporting where the storesman reported that there was no spare part in the stores when it is available and $39 \%$ said there was no loss of business. When the respondents were asked if there was late delivery of service with the current system, 58\% confirmed and attributed this to circumstances where the storesman reports availability of the spare part which is not available while 42 respondents said there was no late delivery of service. When asked if there was pilferage of spares, $76 \%$ said there was pilferage due to lack of effective tracking system of spares while 24 respondents said there was no pilferage. When asked if the introduction of the automated inventory system could mitigate the aforementioned challenges. $91 \%$ of the respondents recommended the introduction of the automated system and $9 \%$ did not recommend the introduction of the automated system. Fig. 9 shows the summary of the results from the baseline study.

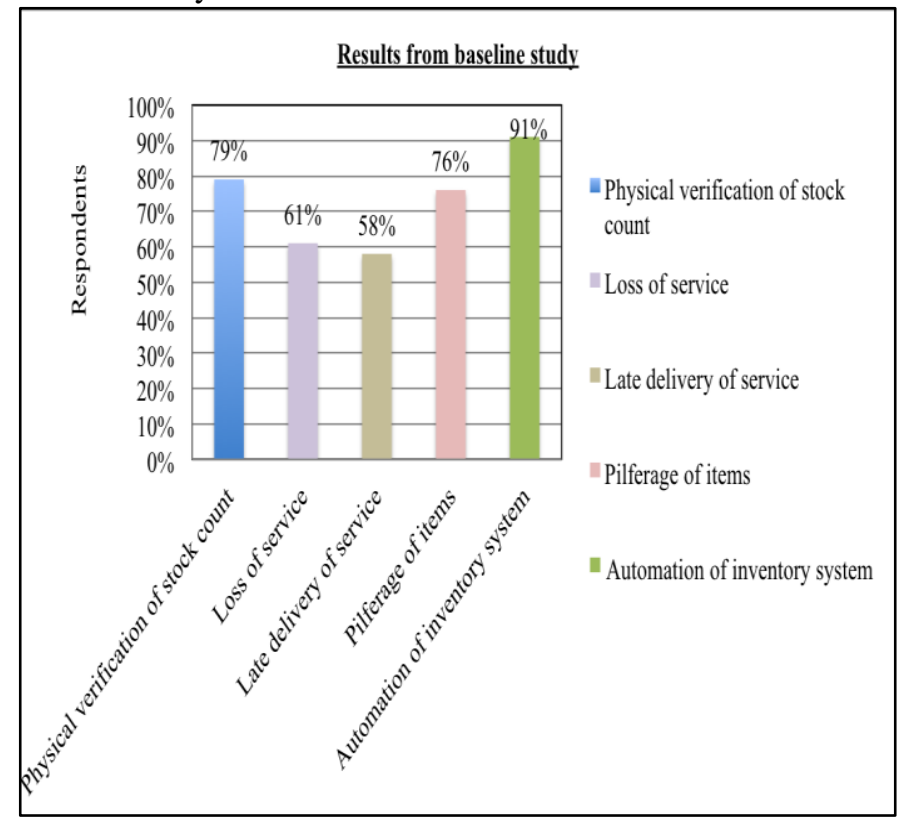

Fig. 9. Baseline study results.

2) System Prototype Development: As already outlined in the previous section, the prototype application named Stock and Inventory Management System (SIMS) consists of the web components which is the frontend and the server-side component which is the backend. The system administrator creates and manages users in the system. The user is registered in the system for them to be able to access the web based system modules. This helps to track every activity performed by specific users in the system. To create a new user account in the system the administrator logs in first and then enters the credentials of the user. Fig. 10 shows the screenshot for the administrator's menu. 


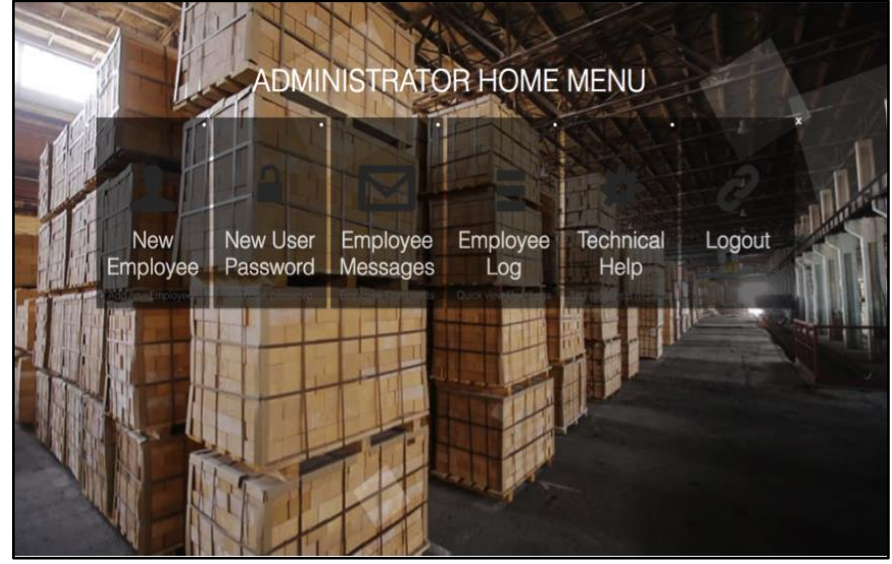

Fig. 10. Administrator Menu.

The administrator can create another administrator and users in the system by giving them user names and default passwords which can later be changed. The passwords are encrypted using MD5 algorithm. Fig. 11 shows the screenshot for registering users.

Fig. 12 shows the login screen for the system, which requires users to provide their usernames and passwords.

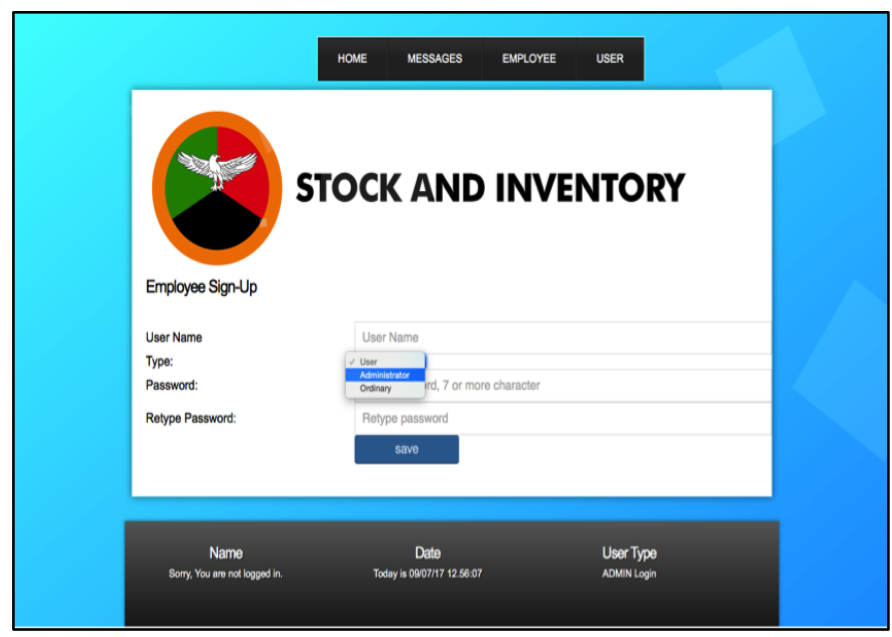

Fig. 11. User registration.

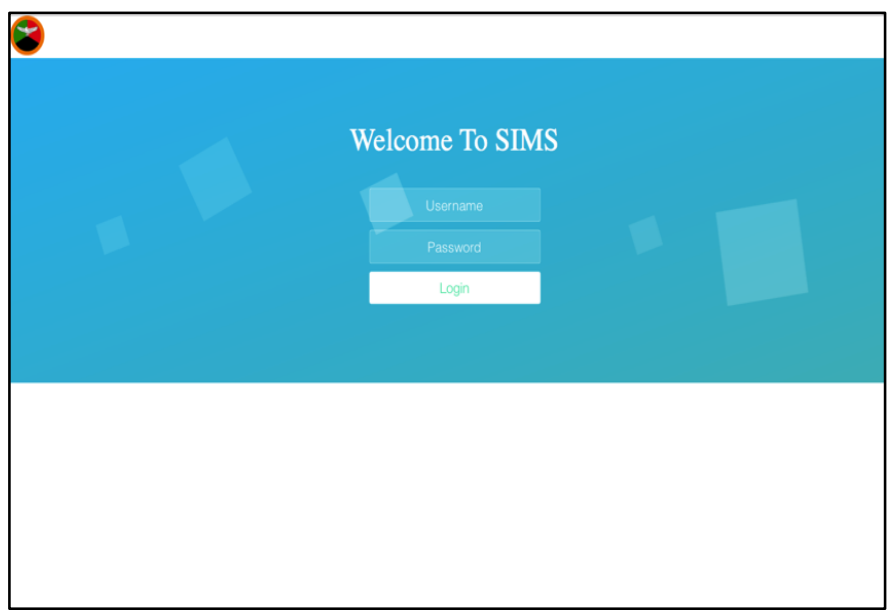

Fig. 12. Login screen.
Fig. 13 shows the main screen. When a user logs in the system, is taken to the main screen. This screen presents a number of options to the user to choose from what task to perform such as add item, add new supplier, transfer item, issue item, print or view reports and many other options.

Fig. 14 and 15 show a web page for adding details of new spares in the system using a barcode reader. The barcode reader scans the barcode on the item and captures the barcode number in the barcode field on the form and then other details related to the item are also entered and written to the database.

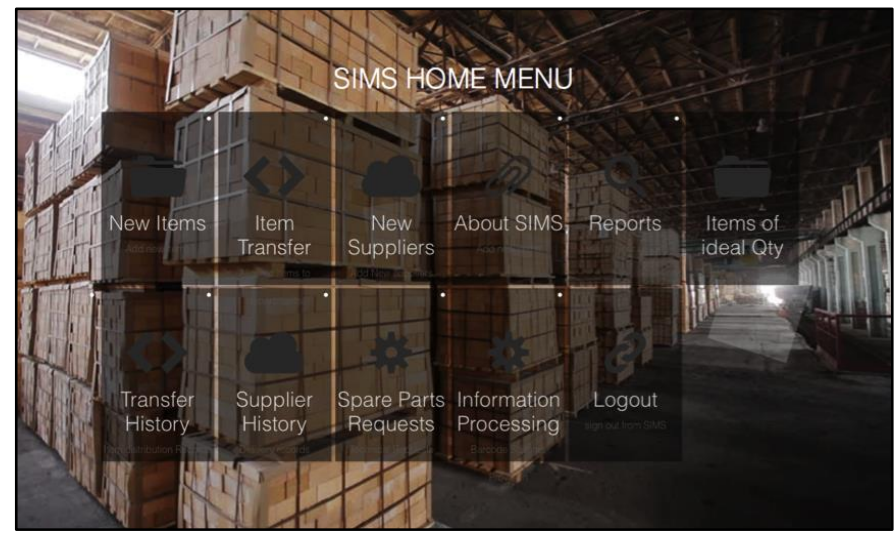

Fig. 13. Main Menu.

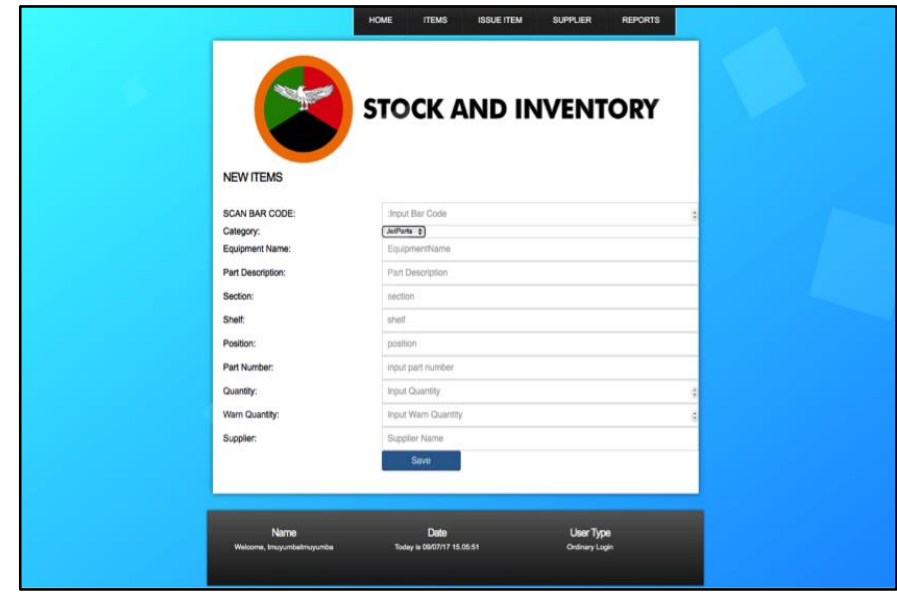

Fig. 14. Adding details of new spares.

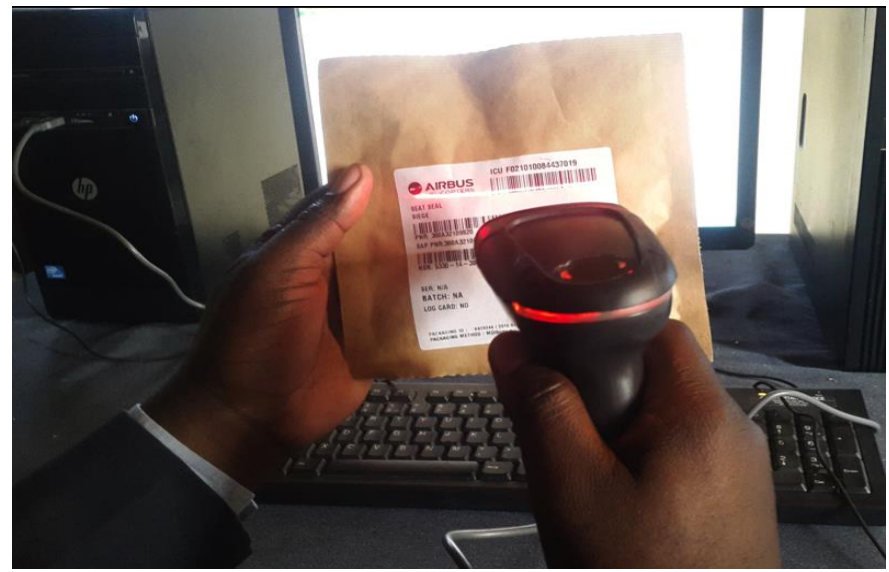

Fig. 15. Adding spares by using barcode reader. 
Fig. 16 shows a web page that is used for issuing out of aircraft spares. When issuing out spares, they are again scanned with the barcode reader. Each spare part that is scanned the quantity reduces in the database. This helps to track the inflow and outflow of spares in the stores.

Fig. 17 shows a screenshot for the results of the report generated for the spare parts that have been issued out. It shows the description of the spare part, category, number of spares issued out, date of issue and the name and rank of the employee who collected the items.

Fig. 18 shows a report of aircraft spares that are below warn quantity.

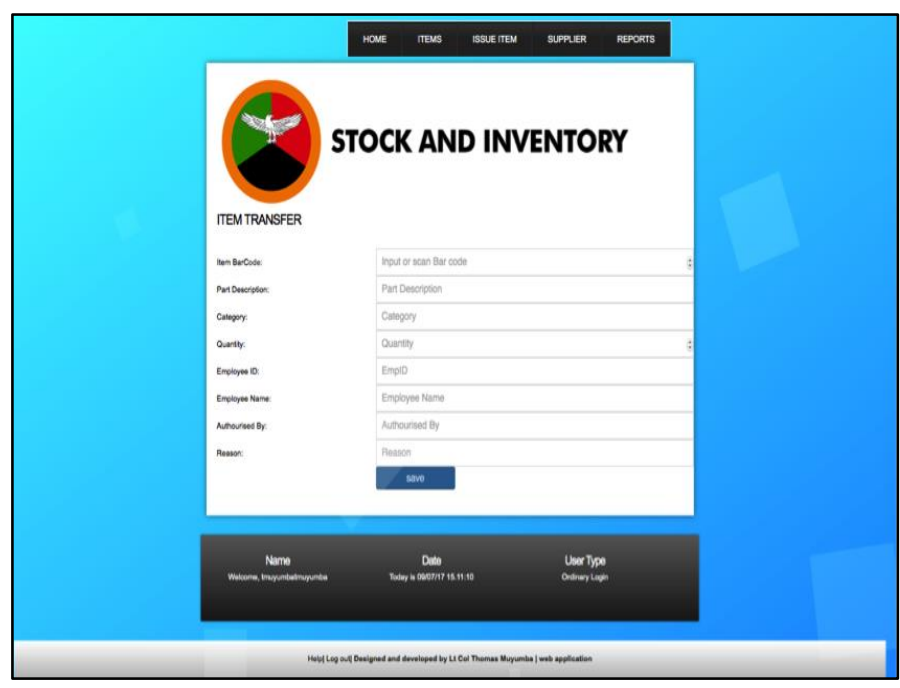

Fig. 16. Issuing spares.

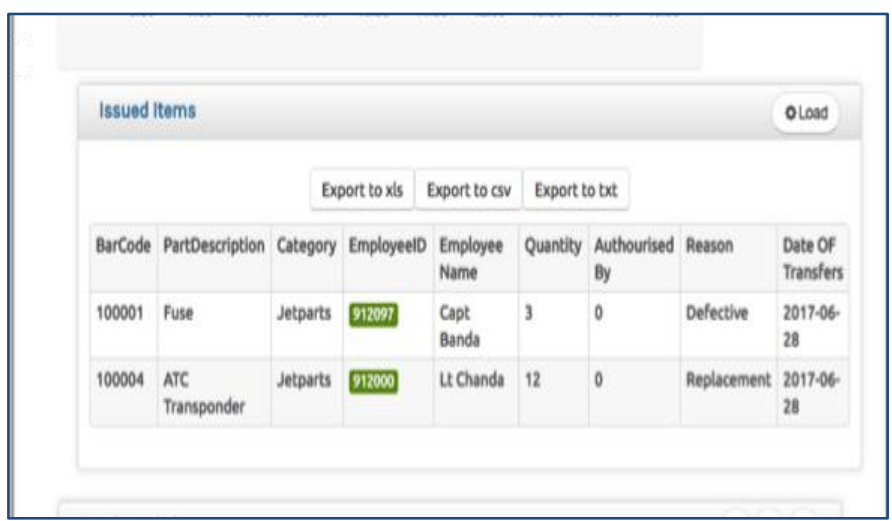

Fig. 17. Issued out spares.

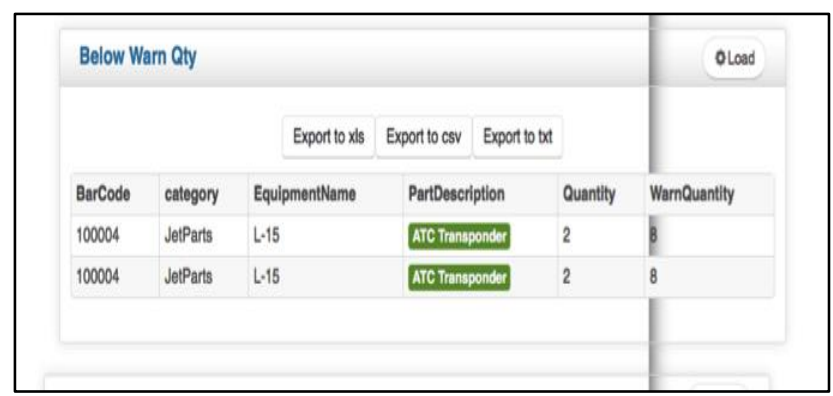

Fig. 18. Below warn quantity.
3) Efficiency Comparison: The one tailed paired t-test was conducted in Microsoft Excel 2013 using the data that was collected showing in Fig. 19 in order to have the scientific evidence of the hypothesis which states that the automated inventory system performs better than the manual and paper based system using the significance level of $\boldsymbol{a}=\mathbf{0 . 0 5}$. Results of the analysis are shown in Fig. 20. Comparing the two inventory systems, the time used by the manual inventory had a significantly higher average time $(\mathrm{M}=41.9, \mathrm{SD}=32.261)$ than the time used by the barcode inventory system $(M=6.4$, $\mathrm{SD}=7.351), \mathrm{t}(9)=(-4.214), \mathrm{P}=0.001$. This result leads to the rejection of the null hypothesis $\left(\mathrm{H}_{0}\right)$ which states that there is no difference in performance between the two systems at the 95\% confidence level, which means that statistically there is sufficient evidence that the barcode inventory is more than 95\% efficient considering the obtained probability value of $\mathbf{p}=$ 0.001.

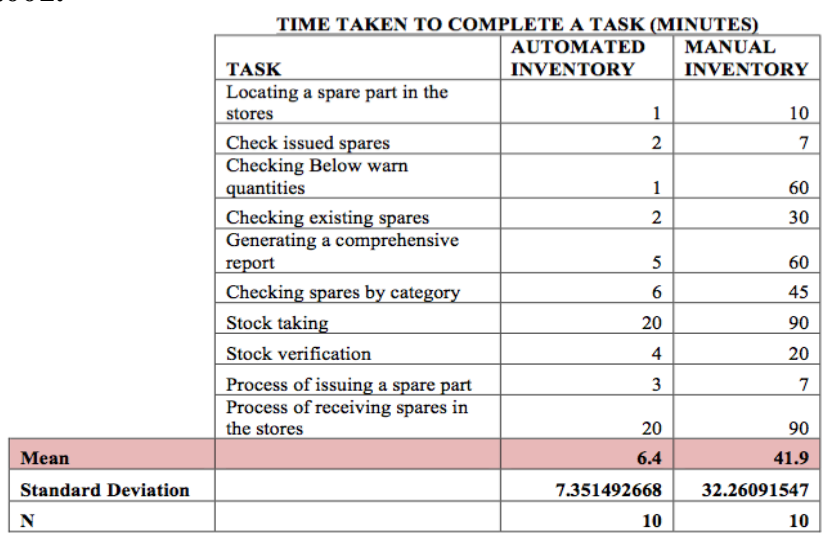

Fig. 19. Collected data.

\begin{tabular}{|c|c|c|}
\hline \multicolumn{3}{|c|}{ t-Test: Paired Two Sample for Means } \\
\hline & AUTOMATED INVENTORY & MANUAL INVENTORY \\
\hline Mean & 6.4 & 41.9 \\
\hline Variance & 54.04444444 & 1040.766667 \\
\hline Observations & 10 & 10 \\
\hline Pearson Correlation & 0.812089822 & \\
\hline Hypothesized Mean Difference & 0 & \\
\hline df & 9 & \\
\hline tStat & -4.214229176 & \\
\hline$P(T<=t)$ one-tail & 0.001129317 & \\
\hline t Critical one-tail & 1.833112933 & \\
\hline$P(T<=t)$ two-tail & 0.002258635 & \\
\hline tCritical two-tail & 2.262157163 & \\
\hline
\end{tabular}

Fig. 20. T-test analysis results.

\section{DISCUSSION}

The main aims of this study were to establish challenges that ZAF faces regarding inventory management of spares and to design a model for an automated barcode inventory management system. From the baseline study conducted, it was discovered that there were a number of challenges that were identified which are highlighted in the results section. The current business processes were mapped as indicated in Fig. 2 and a model based on cloud architecture and barcode technology was developed as indicated in Fig. 3 in order to 
address the challenges that were discovered in the baseline study. A web based inventory prototype was developed using a model based on cloud architecture and barcode technology to mitigate or resolve the challenges faced by ZAF in inventory management. The prototype was tested locally and remotely via MPLS VPN network in which stores personnel were able to add information of spares to the database, issue out spares, verify inventory and generate reports. The following observations were noted during the testing phase of the system:

- The testing that was conducted showed that the bar code scanner was $95 \%$ more efficient and over three times faster than the current method used to manage the inventory in ZAF. This was evidenced by the paired ttest that was conducted.

- The scanning was extremely reliable, with no errors in any of the tests completed. The study supported the possibility of a better means to track spares of the organization in the warehouses.

- It was discovered that the bar code scanners could become the gap filler needed to provide a means of timely and accurate inventories to commanders.

- To address the challenge of pilferage of items, Closed Circuit Television (CCTV) was implemented. IP Cameras were installed in all the warehouses and linked to the cloud network and monitoring is done by sentries on duty in various control centres located in sentry points. The Network Video Recorder (NVR) records the information that is captured by cameras to make it possible to check on previous incidences.

\section{CONCLUSION}

The baseline study was conducted and a number of challenges were identified in the current system such as late delivery of service, loss of service, pilferage of items, high costs and duplication of inventory data. The current business processes were mapped and a model based on cloud architecture and barcode technology was developed in order to address the challenges that were discovered in the baseline study. With the challenges identified and the overwhelming response of 91 percent who recommended that the inventory process be automated, a web based inventory prototype was developed using barcode technology and cloud model to address the challenges. To fully address the challenge involving pilferage of items, CCTV was integrated in the cloud network in which IP cameras were installed in the warehouses. The barcode web based inventory prototype system was fully appreciated by the users in the organization because of its efficiency and effectiveness as evidenced by the t-test that was conducted.

\section{RECOMMENDATIONS AND FUTURE WORKS}

\section{A. Recommendations}

The study has revealed that the automated inventory system is desirable and therefore this system should be fully implemented in order to realize its full benefits.

\section{B. Future Works}

The proposed future works, which should be done on this system, is to migrate from barcode technology to radio frequency identification technology, integrate bulk SMS technology, automate the entire procurement processes and incorporate a module for inventory management of uniforms as well.

\section{REFERENCES}

[1] A. Acilar and Karamasa, "Factors Affecting the E-Commerce Adoption by Small Hotels: A Comparative Case Study," International Journal of E-Adoption (IJEA), vol. 4, no. 1, pp. 1-10, 2012.

[2] K. A. Sarika and S. R. Imran, " Effective Survey On Two-Dimension Color Barcodes For Mobile Applications," International Journal of Scientific Engineering and Applied Science (IJSEAS), vol. 2, no. 1, pp. 195-198, January 2016.

[3] S. Goel and A. K. Singh," A Secure and Optimal QR Code," International Journal of Engineering Research \& Management Technology, vol. 1, no. 5, pp. 36-43, September 2014.

[4] H. A. Mwansele et al., "Determination of Inventory Control Policies at Urafiki Textile Mills Co Ltd in Dares-Salaam, Tanzania," Business and Economics Journal, vol. 2011, pp. 1-9, April 2011.

[5] T. Mayilsamy and P. E. Kumar, "Implementation of E-Kanban System Design in Inventory Management, "International Journal of Scientific and Research Publications, vol. 4, no. 9, pp. 1-4, September 2014.

[6] Dobler \& Burt. (2006). Purchasing management. (6th Ed.).McGraw hill international Edition.

[7] I. O. Victoria and I. W. Ukpere, "The Impact of Effective Inventory Control Management on Organisational Performance: A Study of 7up Bottling Company Nile Mile Enugu, Nigeria," Mediterranean Journal of Social Sciences, vol. 5, no. 10, pp. 109-118, June 2014.

[8] K.D. Mahajan and P. Pandey, "Application of RFID Technology in Libraries and Role of Librarian."

[9] S. Williams, M. Taylor, J. Irland and A. Mehta, "RFID Technology in Forensic Evidence Management, Assessment of Barriers, benefits and Costs," National Institute of Standards and Technoogy, November 2014.

[10] O. Boyinbode and O. Akinyede, "A RFID based Inventory Control System for Nigerian Supermarkets,"International Journal of Computer Applications (0975 - 8887), vol. 116, no. 7, April 2015.

[11] Adaptalift. (2012). RFID Vs Barcodes: Advantages and disadvantages comparison. Logistics \& Materials Handling Blog. Retrieved 21 May 2017 , from http://www.aalhysterforklifts.com.au/index.php/about/blogpost/rfid_vs_ barcodes_advantages_and_disadvantages_comparison.

[12] L. McCathie and K. Michael, (2004), "The advantages and disadvantages of barcodes and radio frequency identification in supply chain management," School of Information Technology and Computer Science. University of Wollongong.

[13] W. R. S. Aguirre et al., "Automated Laboratory Item-Inventory System with Barcode," International Journal of Emerging Technology and Advanced Engineering, vol. 3, no. 12, December 2013.

[14] S. Jyothi and K. Bhargavi," Classification Of DNA Barcodes Based On Image Processing Techniques: A Study," International Journal of Pharma and Bio Sciences, vol. 7, no. 3, pp. 773-780, Joly 2016.

[15] S. Brokaw, "The Expectations of Quick Response (QR) Codes in Print Media: An Empirical Data Research Anthology," W-L Journal of Undergraduate Research, pp. 1-13, 2012.

[16] D. Gladstein, R. Kakarala and Z. Baharay, "3D Barcodes: Theoretical Aspects and Practical Implementation," The International Society for Optical Engineering (Proceedings of SPIE), February 2015.

[17] N. Garg, "Improving Business Logistics using Barcode Scanners," International Journal of Computer Applications, vol. 50, no.15, pp. 1-5, July 2012.

[18] A. Rahul, G. Krishnan, U. Krishnan and S. Rao, "Near Field Communication (Nfc) Technology: A Survey," International Journal on Cybernetics \& Informatics (IJCI), vol. 4, no. 2, pp. 133-144, 2015. 
[19] M. Pulipati and K. P. Srinivas, "Comparison of Various Short Range Wireless Communication Technologies with NFC," International Journal of Science and Research (IJSR), vol. 2, no. 4, pp. 87-91, 2013.

[20] C. Bajaj, "Near Field Communication," International Journal of Advanced Research in Computer Science and Software Engineering, vol. 4, no. 8, pp. 899-905, August 2014.

[21] K. Sankaridevi and V. Vennila, "Near Field Communication (NFC) Technology in Smart E-Transactions," IJSTE -International Journal of Science Technology \& Engineering, vol. 1, no. 11, pp. 247-251, May 2015.

[22] H. A. Al-Ofeishat and M. Rababah, "Near Field Communication (NFC) IJCSNS International Journal of Computer Science and Network Security, vol.12 no.2, pp. 93-99, February 2012.

[23] R. Iqbal, A. Almad, A. Gillani, "NFC based inventory control system for secure and efficient communication," Computer Engineering and Application, vol. 3, no. 1, pp. 23-33, 2014.

[24] K. Kavitha, "Study on Cloud Computing Model and its Benefits, Challenges," International Journal of Innovative Research Computer and Communication Engineering, vol. 2, no. 1, pp. 24232431, January 2014.
[25] P. Sareen, "Cloud Computing: Types, Architecture, Applications, Concerns, Virtualization and Role of IT Governance in Cloud," International Journal of Advanced Research in Computer Science and Software Engineering, vol. 3, no. 3, pp. 533-538, March 2013.

[26] R. P. Padhy, "Cloud Computing: Security Issues and Research Challenges," International Journal of Computer Science and Information Technology \& Security, vol. 1, no. 2, pp. 136-146, December 2011.

[27] A. Chandrasekharan et al., "Barcode Enabled Event Management System for Logistics and Consumables Management," International Journal of Advanced Research in Computer and Communication Engineering, vol. 2, no. 11, pp. 4273-4277, November 2013.

[28] S. Mathaba, N. Dlodlo, A. Smith and M. Adigun, "The use of RFID and Web 2.0 Technologies to Improve Inventory Management in South African Enterprises," Electronic Journal Information Systems Evaluation, vol. 14, no. 2, pp. 225-241, 2011.

[29] S. M. K. Jamal, A. Omer and A. A. S. Qureshi, "Cloud Computing Solution and Services for RFID Based Supply Chain Management," Scientific Research: Advances in Internet of Things, vol. 3, pp. 79-55, October 2013. 